$\frac{10}{2} \cdot 919800$

UICRL-ID--105439

DE9 1007114

\title{
Deconvolution Using a Neural Network
}

S. K. Lehman

November 15, 1990

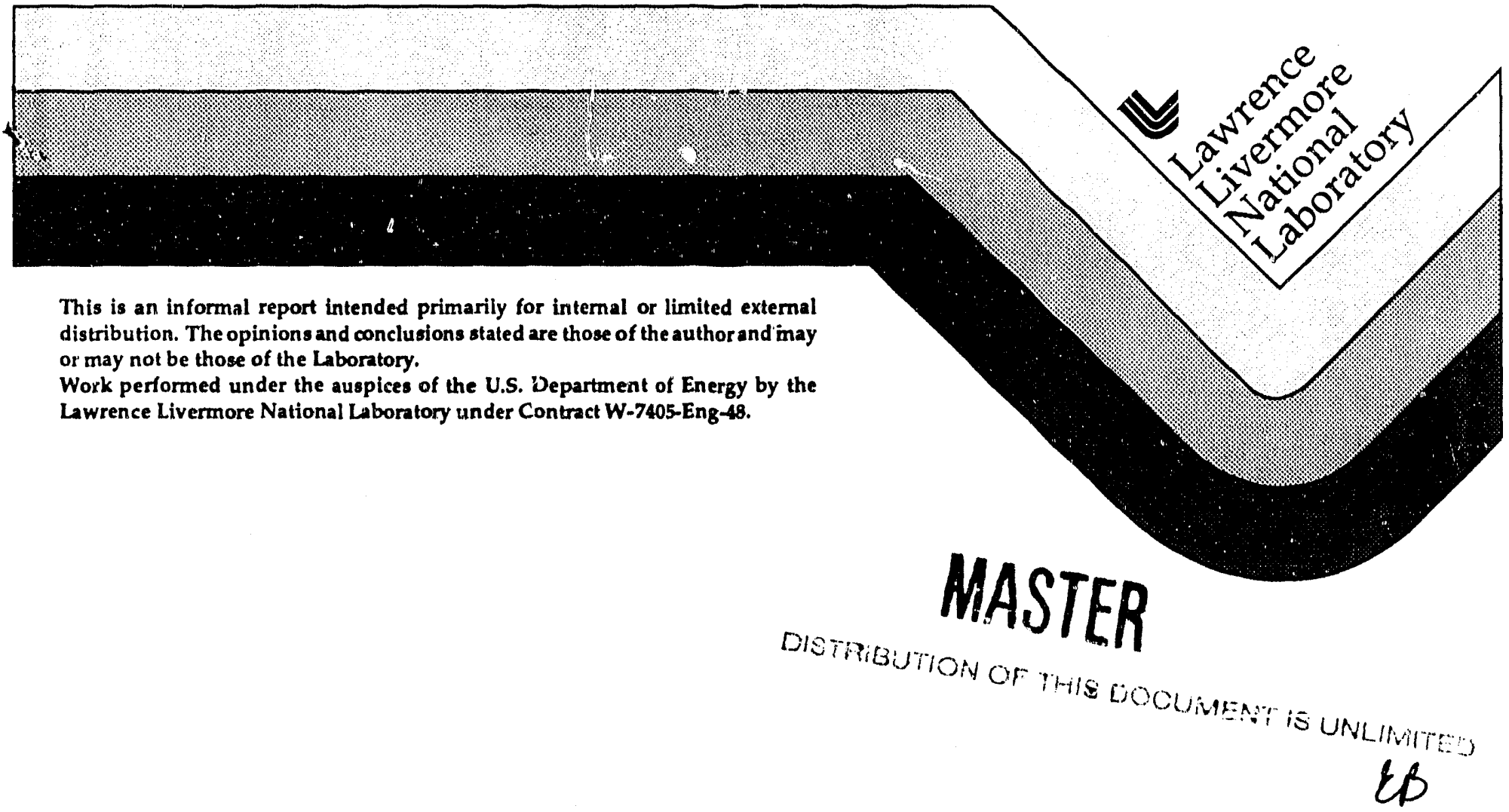


This document was prepared as an account of work sporisored by an agency of the United States Governm. it. Neither the United States Government nor the University of California nor any of their employees, makes any warranky, express or implied, or assumes any legal liability or responsibility for the accuracy, completeness, or usefulness of any information, appalatus, product, or process disclosed, or represents that its use would not infringe privately owned rights. Reference herein to any specific commercial products, process, or service by trade name, trademark, manufacturer, or otherwise, does not necessarily constitute or imply its endorsemer., reccmmendation, or favoring by the United States Government or the University of California. The views and opinions of authors expressed herein do not necessarily state or reflect these of the United States Government or the University of Californis, and shall not be used for advertising or product endorsement purposes.

\author{
This report has been reproduced \\ directly from the best available copy. \\ Available to DOE and DOE contractors from the \\ Office of Scientific and Technical information \\ P.O. Box 62, Cak Ridge, TN 37831 \\ Prices available from 1015) 570-8401, FTS oin-84ill. \\ Available to the public from the \\ National Technical Information Service \\ U.S. Department of Commerce \\ 5285 Porl Royal Rd., \\ Springtield, VA 2216
}

Papercopy rices

$\mathrm{A} 02$

$\mathrm{A03}$

A04

A05

A06

A07

A08

A09

A10

A11

A12

A13

A14

A15

A16

A 17

A 18

A19

A20

A21

A22

A23

A24

A25

A99
1- 10

11- 50

51- 75

76-100

101-125

126-150

151-175

176-200

201-225

226-250

251-275

276-300

301-325

326-350

351-375

376-400

401-425

426-450

451-475

476-500

501-525

526- 550

551-575

576-600

$601 \&$ UP 


\title{
Deconvolution Using a Neural Network*
}

\author{
S. K. Lehman \\ University of California \\ Lawrence Livermore National Laboratory \\ Livermore, CA 94550
}

November 15, 1990

Abstract-Viewing one dimensional deconvolution as a matrix inversion problem, we compare a neural network backpropagation matrix inverse with LMS, and pseudo-inverse. This is largely an exercise in understanding how our neural network code works.

\section{INTRODUCTION}

$\Upsilon$

HE NOISE free convolution of two one-dimensional signals is defined as:

$$
g(n)=\sum_{m=0}^{M-1} f(m) h(n-m)
$$

where $f(m)$, the input signal; $h(k)$, the kernel; and $g(n)$, the result, are defined on

$$
\begin{aligned}
& 0 \leq m \leq M-1 \\
& 0 \leq k \leq K-1 \\
& 0 \leq n \leq M+K-1=N
\end{aligned}
$$

In matrix form:

$$
g=\mathbf{H f}
$$

where $\mathbf{H}$ is the matrix that transforms $\mathbf{f}$ to $\mathrm{g}$ :

$$
\mathbf{H}=\left[\begin{array}{cccccc}
h(0) & 0 & 0 & \cdots & 0 & 0 \\
h(1) & h(0) & 0 & \cdots & 0 & 0 \\
h(2) & h(1) & h(0) & \cdots & 0 & 0 \\
\vdots & \vdots & \vdots & \cdots & 0 & \vdots \\
h(K-2) & h(K-3) & h(K-4) & \cdots & & \\
h(K-1) & h(K-2) & h(K-3) & & h(1) & h(0) \\
0 & h(K-1) & h(K-2) & & h(2) & h(1) \\
0 & 0 & h(K-1) & & h(3) & h^{\prime}(2) \\
\vdots & \vdots & & & \vdots & \vdots \\
0 & 0 & & h(K-1) & h(K-2) & h(K-3) \\
0 & 0 & & 0 & h(K-1) & h(K-2) \\
0 & 0 & \cdots & 0 & 0 & h(K-1)
\end{array}\right]
$$

*Work performct under the auspices of the U. S. Department of Energy by the Lawrence Livermore National Laboratory under contract number W-7405-ENG-48. 
The problem is to find $\mathbf{A}$ such that

$$
\mathbf{f}=\mathbf{A g}
$$

We are also interested in the convolution problem. That is finding $\mathbf{H}$ such that

$$
\mathbf{g}=\mathbf{H f}
$$

We compare three methods for determining either $\mathbf{H}$ given $\mathbf{A}$; or $\mathbf{A}$ given $\mathbf{H}$; or $\mathbf{H}$ and $\mathbf{A}$ given an appropriate set of fs and gs. The methods are:

1. Pseudo-inverse ;

2. Neural network ;

3. LMS.

\section{Pseudo-inverse}

Given $\mathbf{H}, \mathbf{A}$ is determined by:

$$
\begin{aligned}
\mathbf{H}^{\top} \mathbf{g} & =\mathbf{H}^{\top} \mathbf{H} \mathbf{f} \\
\left(\mathbf{H}^{\top} \mathbf{H}\right)^{-1} \mathbf{H}^{\top} \mathbf{g} & =\mathbf{f} \\
\mathbf{A g} & =\mathbf{f}
\end{aligned}
$$

Where

Similarly, given $\mathbf{A}$ :

$$
\mathbf{A}=\left(\mathbf{H}^{\top} \mathbf{H}\right)^{-1} \mathbf{H}^{\top}
$$

$$
\mathbf{H}=\left(\mathbf{A}^{\top} \mathbf{A}\right)^{-1} \mathbf{A}^{\top}
$$

\section{NeUral Network}

To determine $\mathbf{H}$, we use a one layer network with $M$ inputs and $M+K-1$ outputs and train on a set of impulses and their responses. There are $2 M$ elements in the set since we have to train on negative as well as the positive impulses. (When trained using only the positive impulses, the netwuik converges but not correctly.)

Once converged, the weights make up $\mathbf{H}$. Because this is a linear problem the biases are all rero.

To determine $\mathbf{A}$, we use a network with $M+K-1$ inputs and $M$ outputs. We use the sanie training set as above except the impulse responses are the inputs and impulses are the targets.

Note in both networks we do not use threshold functions.

\section{LMS}

Given a set of input $\left\{\mathbf{f}_{\mathbf{i}}\right\}$ and output $\left\{\mathbf{g}_{\boldsymbol{i}}\right\}$ data determine $\mathbf{H}$.

For the derivation of the LMS solution we temporarily drop the vector index $i$ since we use tensor notation and do not want to confuse the it with the vector element index.

The goal is to minimize:

$$
\begin{aligned}
\mathcal{E}^{2} & =(\mathbf{g}-\mathbf{H f})^{\top}(\mathbf{g}-\mathbf{H f}) \\
& =\left(\mathbf{g}^{\top}-\mathbf{f}^{\top} \mathbf{H}^{\top}\right)(\mathbf{g}-\mathbf{H} \mathbf{f}) \\
& =\mathbf{g}^{\top} \mathbf{g}-2 \mathbf{g}^{\top} \mathbf{H} \mathbf{f}+\mathbf{f}^{\top} \mathbf{H}^{\top} \mathbf{H} \mathbf{f}
\end{aligned}
$$

In tersor notation:

$$
\mathcal{E}^{2}=\mathrm{g}_{i} \mathrm{~g}_{i}-2 \mathrm{~g}_{i} \mathrm{H}_{i j} \mathrm{f}_{j}+\mathrm{f}_{i} \mathrm{H}_{j i} \mathrm{H}_{j k} \mathrm{f}_{k}
$$

Where $g_{i}$ is the $i^{\text {th }}$ component of the vector $\mathbf{g}$ and not the $i^{\text {th }}$ vector in the set $\left\{g_{i}\right\}$. 


$$
\begin{aligned}
\frac{\partial \mathcal{E}^{2}}{\partial \mathrm{H}_{m n}} & =-2 \mathrm{~g}_{i} \frac{\partial \mathrm{H}_{i j}}{\partial \mathrm{H}_{m n}} \mathrm{f}_{j}+\mathrm{f}_{i} \frac{\partial\left[\mathrm{H}_{j i} \mathrm{H}_{j k}\right]}{\partial \mathrm{H}_{m n}} \mathrm{f}_{k} . \\
& =-2 \mathrm{~g}_{i} \delta_{i m} \delta_{j n} \mathrm{f}_{j}+\mathrm{f}_{i}\left[\delta_{j m} \delta_{i n} \mathrm{H}_{j k}+\delta_{j m} \delta_{n k} \mathrm{H}_{j i}\right] \mathrm{f}_{k} \\
& =-2 \mathrm{~g}_{m} \mathrm{f}_{n}+\mathrm{f}_{i}\left[\delta_{i n} \mathrm{H}_{m k}+\delta_{n k} \mathrm{H}_{m i}\right] \mathrm{f}_{k} \\
& =-2 \mathrm{~g}_{m} \mathrm{f}_{n}+\mathrm{f}_{n} \mathrm{H}_{m k} \mathrm{f}_{k}+\mathrm{f}_{i} \mathrm{H}_{m i} \mathrm{f}_{n} \\
& =-2 \mathrm{~g}_{m} \mathrm{f}_{n}+\mathrm{f}_{n} \mathrm{H}_{m k} \mathrm{f}_{k}+\mathrm{f}_{n} \mathrm{H}_{m i} \mathrm{f}_{i} \\
& =-2 \mathrm{~g}_{m} \mathrm{f}_{n}+2 \mathrm{f}_{n} \mathrm{H}_{m k} \mathrm{f}_{k}
\end{aligned}
$$

Back to matrix notation:

$$
\frac{\partial \mathcal{E}^{2}}{\partial \mathbf{H}}=-2 \mathbf{g f}^{\top}+2 \mathbf{H} \mathbf{f f}^{\top}
$$

Summing over the data set, $\left\{\mathbf{f}_{i}\right\}$ and $\left\{\mathbf{g}_{i}\right\}$, and equating to zero:

$$
\begin{gathered}
\sum_{i} \frac{\partial \mathcal{E}_{i}^{2}}{\partial \mathbf{H}}=-2 \sum_{i} \mathbf{g}_{i} \mathbf{f}_{i}^{\top}+2 \mathbf{H}\left[\sum_{i} \mathbf{f}_{i} \mathbf{f}_{i}^{\top}\right]=0 \\
\Rightarrow \mathbf{H}=\left[\sum_{i} \mathbf{g}_{i} \mathbf{f}_{i}^{\top}\right]\left[\sum_{i} \mathbf{f}_{i} \mathbf{f}_{i}^{\top}\right]^{-1}
\end{gathered}
$$

In a similar manner we may determine $\mathbf{A}$ :

$$
A=\left[\sum_{i} f_{i} g_{i}^{\top}\right]\left[\sum_{i} g_{i} g_{i}^{\top}\right]^{-1}
$$

\section{REsults}

For a detailed transcript of the computer results see the appendices. They present a session training the neural network to deconvolve, a session training it to convolve, and a Mathematica ${ }^{\mathrm{TM}}$ [1] session used to verify the results.

As our test case we use:

$$
\begin{aligned}
& M=5 \\
& K=3 \\
& N=7 \\
& h(k)=\left\{\begin{array}{lll}
1 & -1 & 1
\end{array}\right\} \\
& \Rightarrow \mathbf{H}=\left[\begin{array}{rrrrr}
1 & 0 & 0 & 0 & 0 \\
-1 & 1 & 0 & 0 & 0 \\
1 & -1 & 1 & 0 & 0 \\
0 & 1 & -1 & 1 & 0 \\
0 & 0 & 1 & -1 & 1 \\
0 & 0 & 0 & 1 & -1 \\
0 & 0 & 0 & 0 & 1
\end{array}\right]
\end{aligned}
$$


The set of impulses and their responses used in training are:

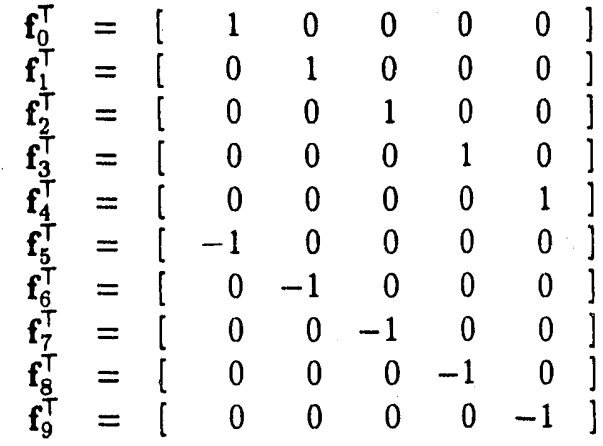

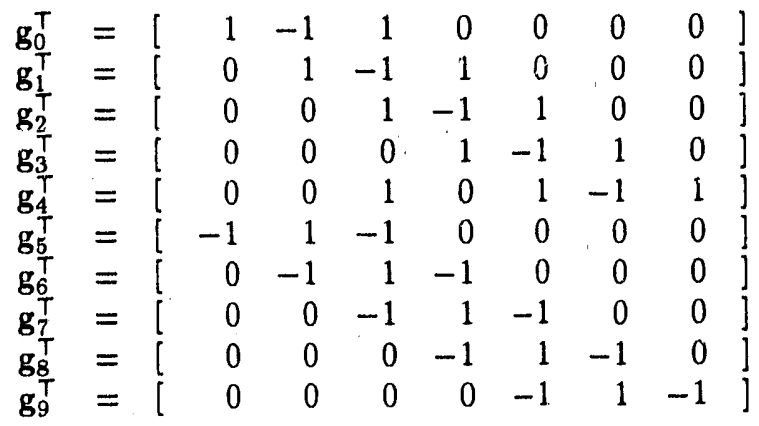

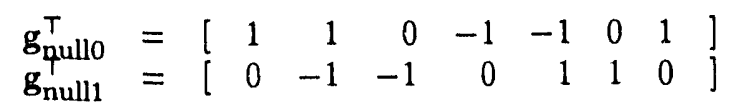

Using Mathematica ${ }^{\mathrm{TM}}$, we used (6) to compute $\mathbf{A}$ from (14). We then verified $\mathbf{A}_{\text {math }} \cdot \mathbf{H}=\mathbf{I}$ and $\mathbf{A}_{\text {math }} \mathbf{g}_{\boldsymbol{i}}=\mathbf{f}_{\boldsymbol{i}}$. This is the pseudo-inverse solution from Mathematica ${ }^{\mathrm{TM}}$ :

$$
\mathbf{A}_{\text {math }}=\left[\begin{array}{ccccccc}
3 / 4 & -1 / 8 & 1 / 8 & 1 / 4 & 1 / 8 & -1 / 8 & -1 / 4 \\
5 / 8 & 9 / 16 & -1 / 16 & 3 / 8 & 7 / 16 & 1 / 16 & -3 / 8 \\
0 & 1 / 2 & 1 / 2 & 0 & 1 / 2 & 1 / 2 & 0 \\
-3 / 8 & 1 / 16 & 7 / 16 & 3 / 8 & -1 / 16 & 9 / 16 & 5 / 8 \\
-1 / 4 & -1 / 8 & 1 / 8 & 1 / 4 & 1 / 8 & -1 / 8 & 3 / 4
\end{array}\right]
$$

When we applied (7) to (18) to get $\mathbf{H}$ back again, we got a Mathematica ${ }^{\mathrm{TM}}$ error saying $\mathbf{A}_{\text {math }}^{\top} \mathbf{A}_{\text {math }}$ was singular.

The neural network we trained to convolve yielded (14) exactly. However, the network we trained to deconvolve, found a different $\mathbf{A}$ than the pseudo-inverse method:

$$
\mathbf{A}_{n n}=\left[\begin{array}{rrrrrrr}
0.4049 & -0.2637 & 0.3314 & 0.5951 & 0.2637 & -0.3314 & -0.5951 \\
0.5227 & 0.6245 & 0.1018 & 0.4773 & 0.3755 & -0.1018 & -0.4773 \\
0.1600 & 0.4017 & 0.2417 & -0.1600 & 0.5983 & 0.7583 & 0.1600 \\
-0.1343 & 0.0583 & 0.1926 & 0.1343 & -0.0583 & 0.8074 & 0.8657 \\
-0.2143 & -0.2585 & -0.0442 & 0.2143 & 0.2585 & 0.0442 & 0.7857
\end{array}\right]
$$

We verified $\mathbf{A}_{n n} \cdot \mathbf{H}=\mathbf{I}$ and $\mathbf{A}_{n n} \mathbf{g}_{i}=\mathbf{f}_{i}$. We entered (19) into Mathematica ${ }^{\mathrm{TM}}$ and applied (6) to it but got the singular matrix error.

We applied (11) in Mathematica ${ }^{\mathrm{TM}}$ to the data set, $\left\{\mathbf{f}_{i}\right\}$ and $\left\{\mathbf{g}_{i}\right\}$, we presented to the neural network. It computed $\mathbf{H}$ exactly. However (12) resulted in a singular matrix error when Mathematica ${ }^{\mathrm{TM}}$ tried to invert $\sum_{i} g_{i} \mathbf{g}_{i}^{\top}$. This is because half of the $\left\{g_{i}\right\}$ are linearly dependent.

The $\left\{\mathbf{f}_{i}\right\}$ span an $M$-space. The $\left\{\mathbf{g}_{i}\right\}$ span an $M$-dimensional subspace of an $N$-dimensional space. Recall $M<N$. 
H maps an $M$-space to an $N$-space. Since we have $M(=5)$ linearly independent $\left\{\mathbf{f}_{i}\right\}$, we can use (11) to find $\mathbf{H}$. However in finding the mapping, $\mathbf{A}$, from a 7-space to a 5-space, we have only 5 independent $\left\{g_{i}\right\}$, thus (12) will not work. It needs more information. The neural network somehow "guesses" at the additional information in order to find the inverse.

The solution the neural network finds is not the minimum norm solution. Equation (18) is the minimum norm solution since the pseudo-inverse is the LMS solution. The solution, $\mathbf{A}_{n n}$, the neural network finds can be written as:

$$
\mathbf{A}_{n n}=\mathbf{A}_{\|}+\mathbf{A}_{\perp}
$$

where $\mathbf{A}_{\|}\left(=\mathbf{A}_{\text {math }}\right)$ is parallel to the 5-subspace spanned by $\left\{\mathbf{g}_{i}\right\}$ and $\mathbf{A}_{\perp}$ is perpendicular to it. The minimum norm solution is $\mathbf{A}_{\|}$. By subtracting (18) from (19), $\mathbf{A}_{n n}-\mathbf{A}_{\text {math }}$, we obtained an $\mathbf{A}_{\perp}$. We verified in Mathematica ${ }^{\mathrm{TM}}$ that $\mathbf{A}_{\perp} \cdot \mathbf{g}_{i}=\mathbf{0}$.

We used Mathematica ${ }^{\text {TM }}$ to find the null-space vectors of $\mathbf{A}_{\text {math }}$, equation (17), and included them with the set of vectors used in training the neural network to deconvolve. The resulting weights were (18) exactly.

Note $\mathbf{A}_{\|}$is the best solution for noise elimination since the noise would span the full 7-space.

\section{Conclusions}

A feed forward, backpropagation trained, one layer neural network with no thresholding functions can be trained to convolve and deconvolve noise free data.

When given identical vectors set, the neural network was able to find the convolution matrix whereas the LMS method was not.

\section{ACKNOWLEDGMENTS}

The author wishes to acknowledge the help and encouragement of J. P. Fitch and D. T. Gavel.

\section{REFERENCES}

[1] Stephen Wolfram, Mathematica, A System for Doing Mathematics by Computer, Addison-Wesley Publishing Company, NY 1988.

\section{A Deconvolving Network}

This is the shell script used in training the network to deconvolve. The case presented includes the two null-space vectors, thus the $\mathbf{A}$ found is that of the LMS solution.

First the training code, learn, is called, followed by apply, the testing code and then disect which "disects" the network to examine the weights.

For a detailed description on code operation contact the author.

$\# ! / \mathrm{bin} / \mathrm{sh}$

Train=dtraino-null

Test $=d t e s t$

Rang $\theta=1.0 \theta 12$

Err $=1 . \theta-10$

if ( test $-z$ "\$1") then Method=1; else Method=\$1; Ii

set 'wc - 1 \$rain \$Test'

Eleml $=\$ 1$

Elemt $=\$ 3$ 


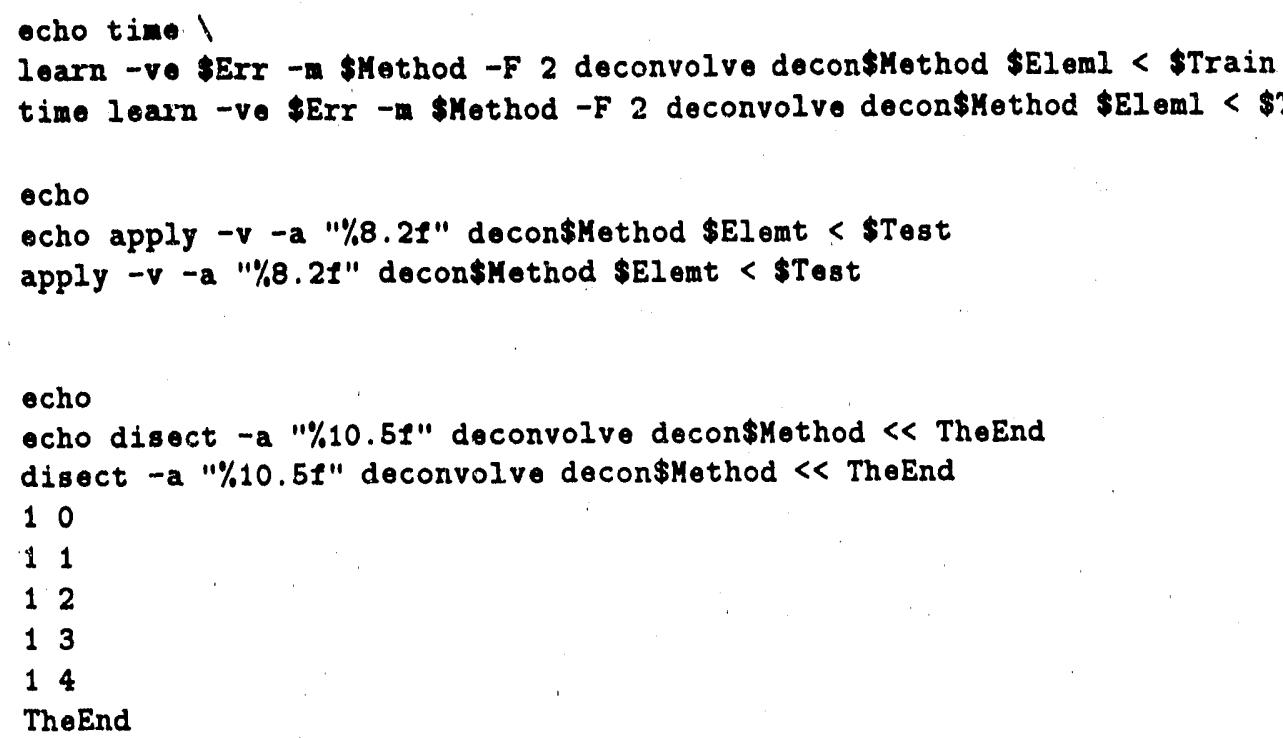

This is a typescript of the execution of the above shell script.

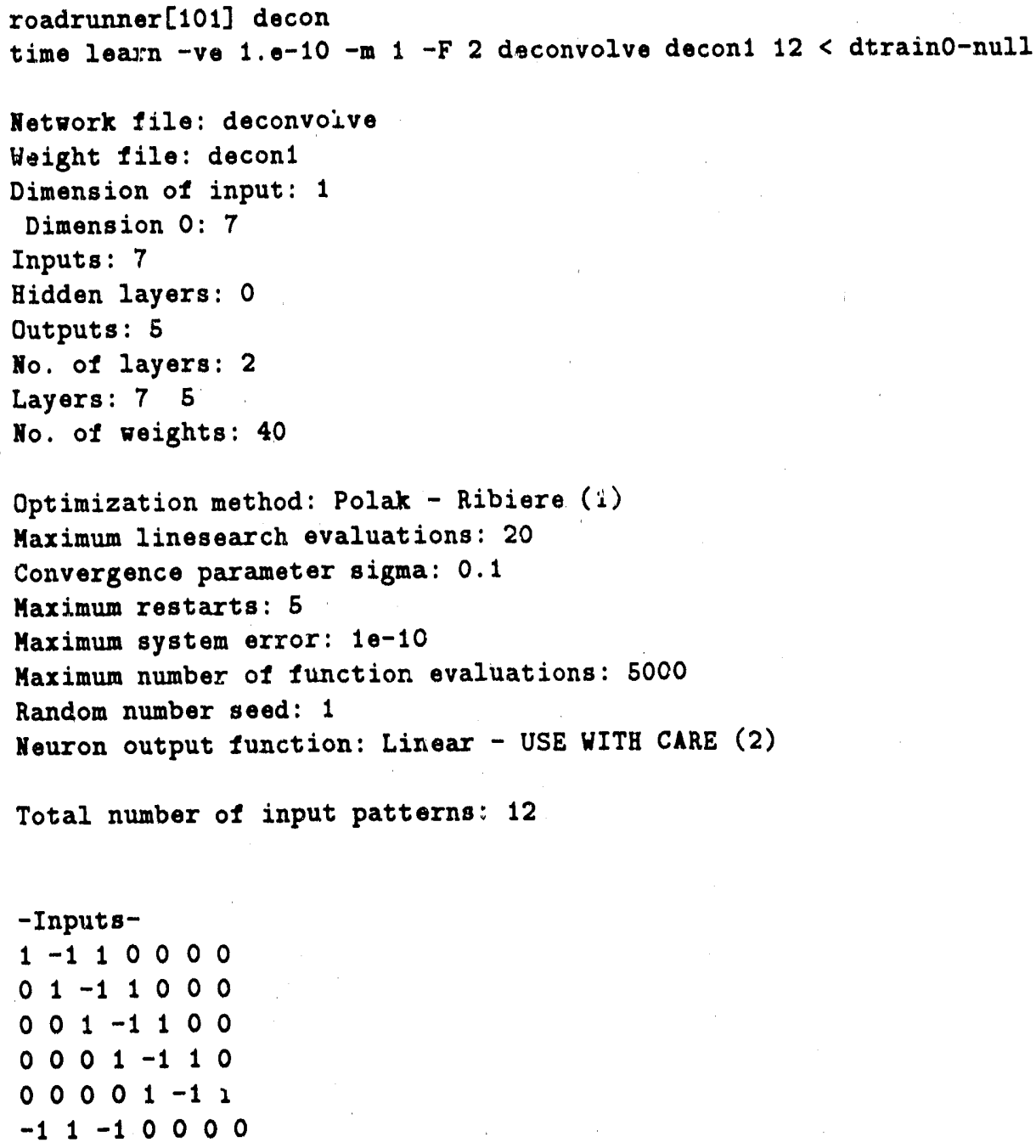




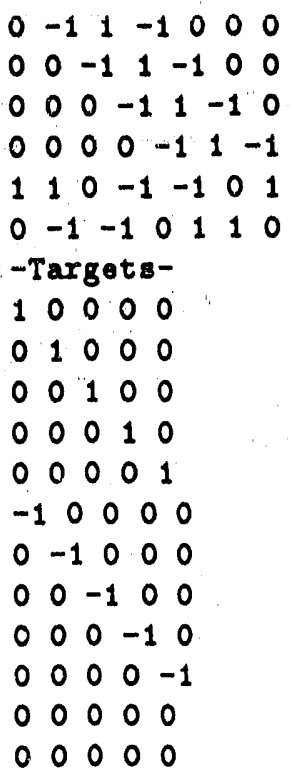

Error at start: 0.91579, gnorm $=1.08015$

Iteration 1, error $=0.277851$, gnorm $=$

0.375408

Iteration

2 , error $=$

0.13769 , gnorm $=$

0.273439

Iteration

3 , error $=$

0.0555376 , gnorm $=$

0.178131

Iteration

4, orror $=$

0.0246831 , gnorm $=$

0.109281

Iteration

5, ',rror =

0.0060042 , gnorm $=$

0.0578176

Iteration

6 , error =

0.0015934 , gnorm $=$

0.0214331

Iteration

7 , error $=$

0.000140781 , gnorm $=$

0.00510232

Iteration

8 , error $=4.20221 \mathrm{e}-28$, gnorm $=3.222 .14 \mathrm{e}-14$

Total number of iterations is 8

Total number of functicn evaluations is 18

Normalized systew error. is $4.20221 \theta-28$

Horm of the gradient is $3.22214 \theta-14$

Maximum pattern error is $9.08972 \theta-28$

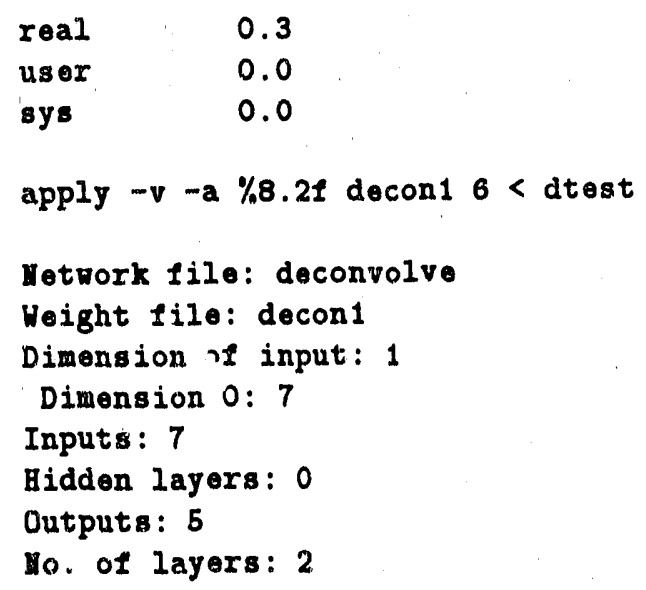


Layers: 76

No. of woights: 40

\begin{tabular}{|c|c|c|c|c|c|}
\hline \multicolumn{6}{|c|}{-Inputs- } \\
\hline \multicolumn{6}{|c|}{$\begin{array}{lllllll}0 & 1 & -1 & 0 & 1 & -1 & 0\end{array}$} \\
\hline \multicolumn{6}{|c|}{1011101} \\
\hline \multicolumn{6}{|c|}{$\begin{array}{lllllll}0 & 1 & 1 & 2 & 3 & -1 & 4\end{array}$} \\
\hline \multicolumn{6}{|c|}{0110110} \\
\hline \multicolumn{6}{|c|}{$\begin{array}{llllllll}1 & -2 & 3 & -3 & 3 & -2 & 1\end{array}$} \\
\hline \multicolumn{6}{|c|}{$\begin{array}{lllllll}5 & -10 & 15 & -15 & 15 & -10 & 5\end{array}$} \\
\hline \multicolumn{6}{|c|}{-Outputs- } \\
\hline 1) & 0.00 & 1.00 & 0.00 & -1.00 & 0.00 \\
\hline 2) & 1.00 & 1.00 & 1.00 & 1.00 & 1.00 \\
\hline 3) & 0.00 & 1.00 & 2.00 & 3.00 & 4.00 \\
\hline 4) & 0.00 & 1.00 & 2.00 & 1.00 & 0.00 \\
\hline 5) & 1.00 & -1.00 & 1.00 & -1.00 & 1.00 \\
\hline 6) & 5.00 & -5.00 & 5.00 & -5.00 & 5.00 \\
\hline
\end{tabular}

disect $-a \% 10.51$ deconvolve decon $1 \ll$ TheEnd

Metrork file: deconvolve

Weight file: decon 1

Dimension of input: 1

Dimension 0: 7

Inputs : 7

Hidden layers: 0

Outputs: 5

Ho. of layers: 2

Layers: 75

Ho. of veights: 40

Enter disection layer neuron ( $D$ to quit):

Dimension of sample: 1

Dimension $0: 7$

Layer: 1

Heuron: 0

Bias: $1.89735 \theta-16$
$0.75000-0.12500$
0.12500
0.25000
$0.12500-0.12500-0.25000$

Layer neuron ( $D$ to quit):

Dimension of sample: 1

Dimension 0: 7

Lay $0 x$ : 1

Heuron: 1

Bias: $5.88234 e-15$
0.62500
$0.56250-0.06250$
0.37500
0.43750
$0.06250-0.37500$ 


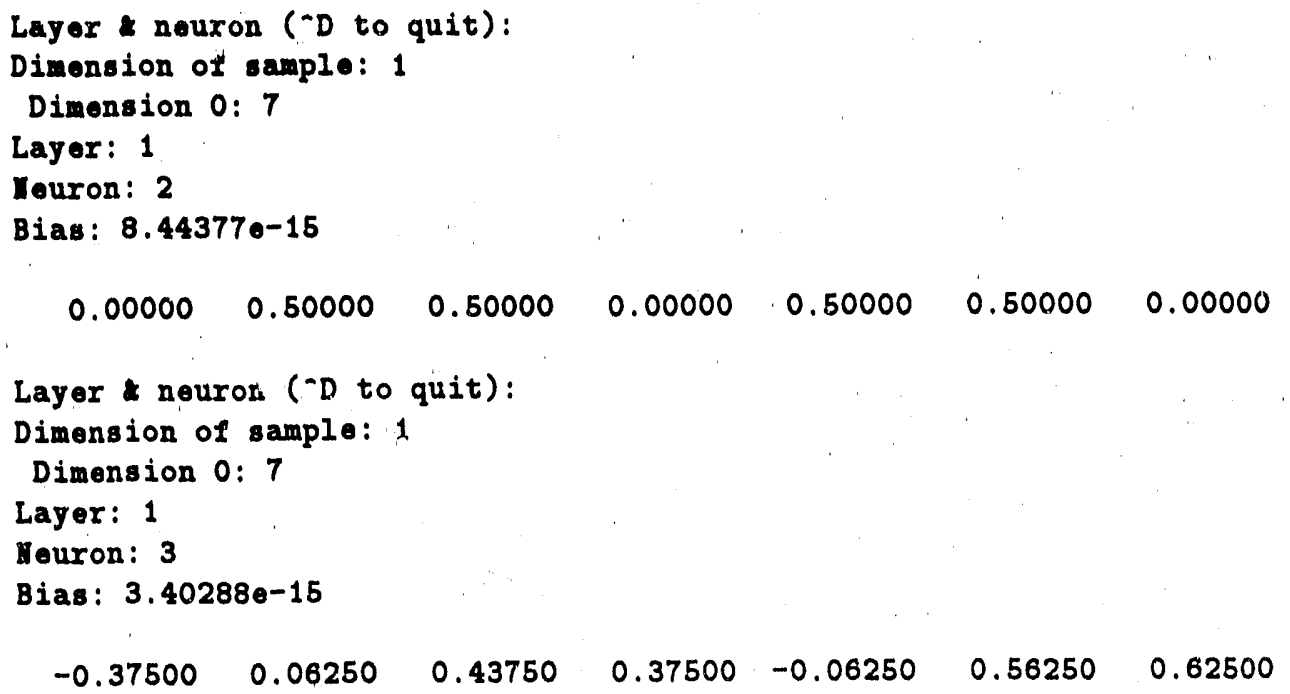

\section{B Convolving Network}

This is the shell script used in training a network to convolve. It is identical in form to the deconvolution shell script.

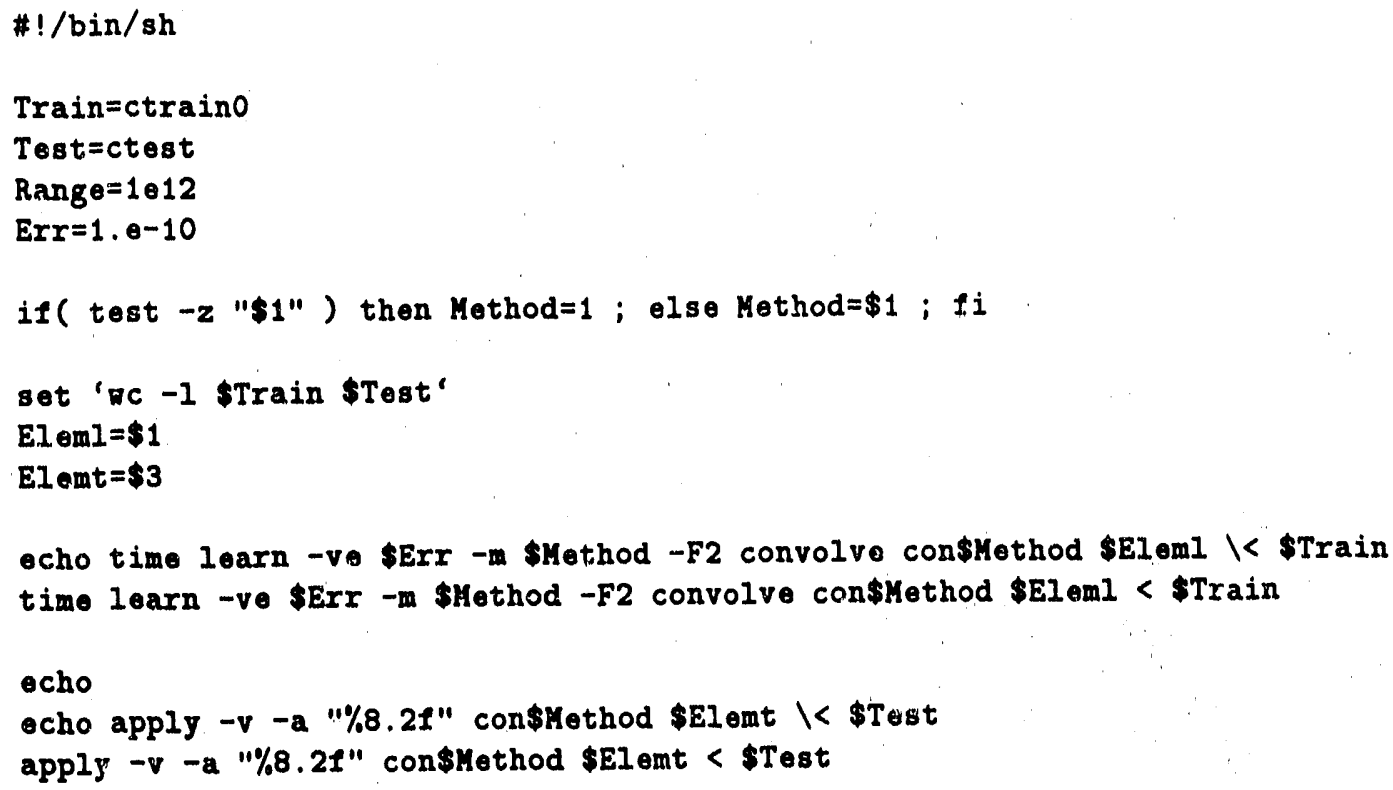




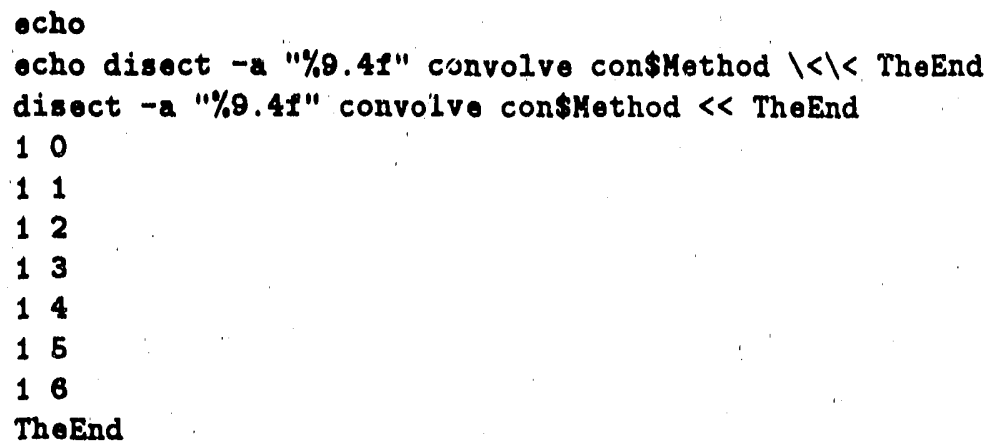

12

13

14

15

16

The End

This is a typescript of the execution of the above shell script:

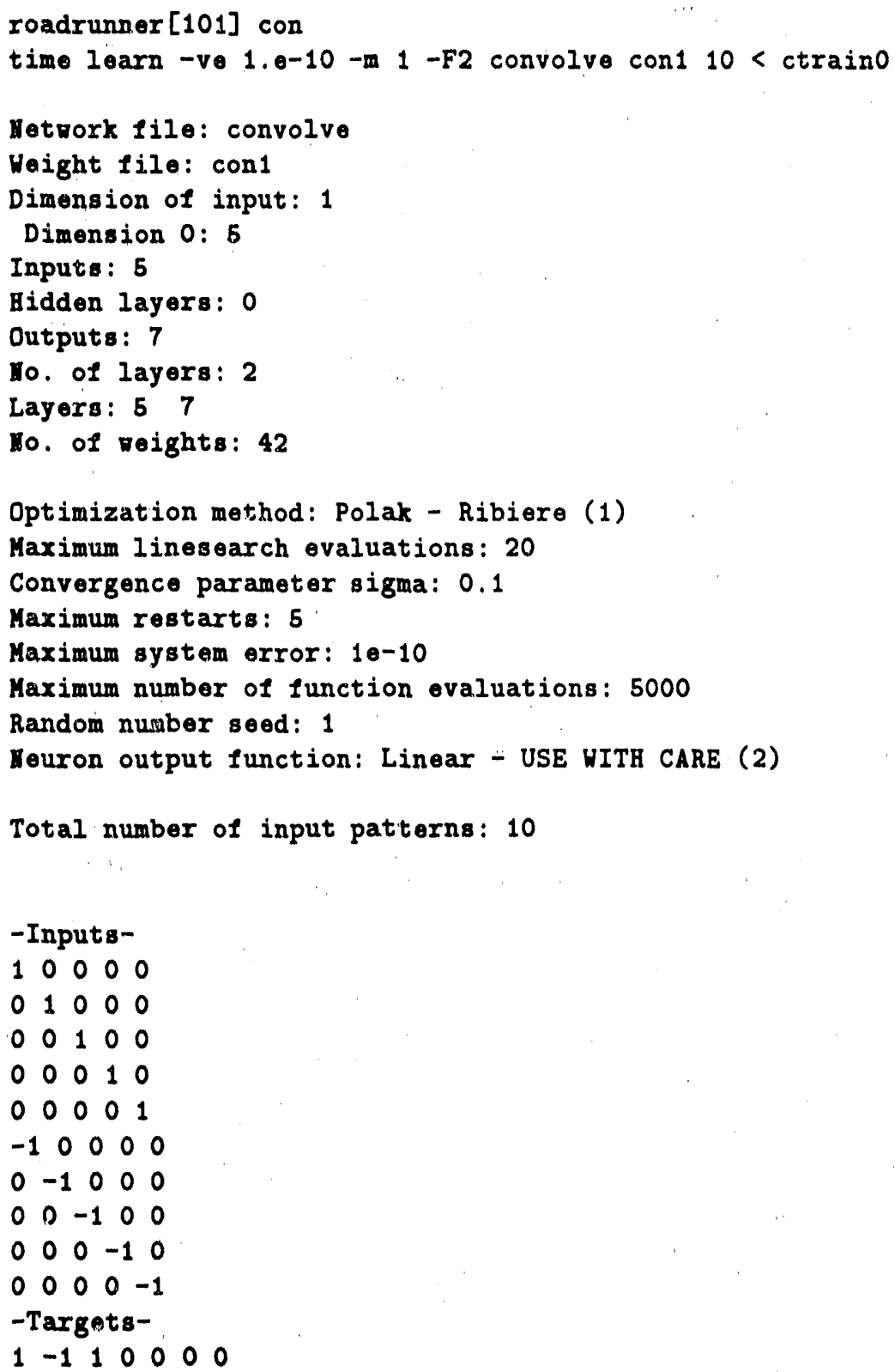




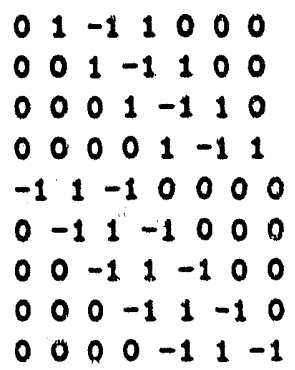

Error at start: 2.45993, gnorm $=1.27743$

Iteration 1 , error $=1.09327$, gnom $=0.855856$

Iteration 2 , exror $=1.107710-31$, gnorm $=4.233880-16$

Total number of iterations is?

Total number of function craluations is 6

Yormalized systen orror is 1.107710-31

lore of the gradient is $4.233880-16$

Maximul pattern error is $1.722740-31$

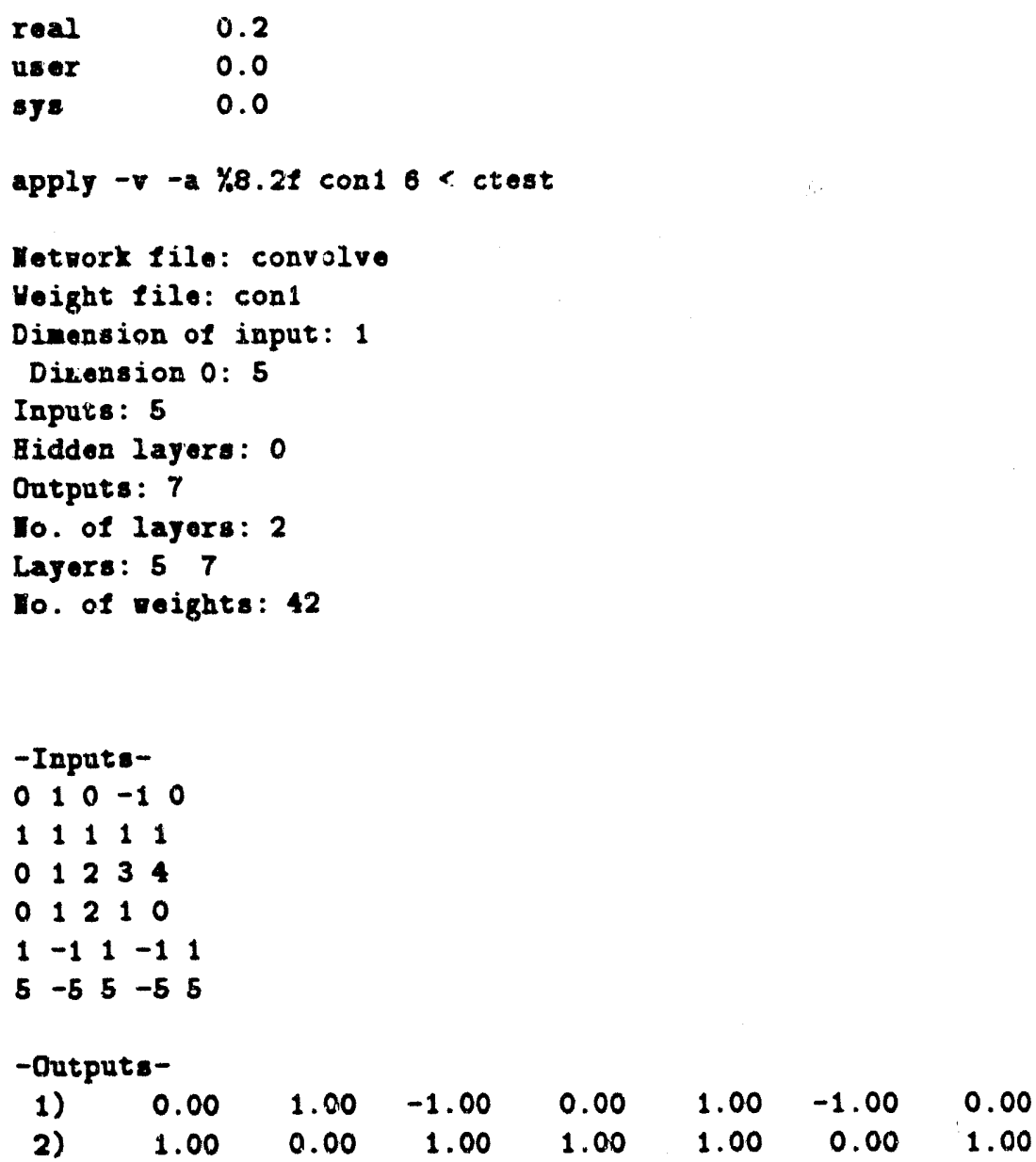

$\begin{array}{lrrrrrrr}\text { 1) } & 0.00 & 1.00 & -1.00 & 0.00 & 1.00 & -1.00 & 0.00 \\ \text { 2) } & 1.00 & 0.00 & 1.00 & 1.00 & 1.00 & 0.00 & 1.00\end{array}$




$\begin{array}{lrrrrrrr}3) & 0.00 & 1.00 & 1.00 & 2.00 & 3.00 & -1.00 & 4.00 \\ \text { 4) } & 0.00 & 1.00 & 1.00 & 0.00 & 1.00 & 1.00 & 0.00 \\ \text { 5) } & 1.00 & -2.00 & 3.00 & -3.00 & 3.00 & -2.00 & 1.00 \\ \text { 6) } & 6.00 & -10.00 & 15.00 & -15.00 & 15.00 & -10.00 & 5.00\end{array}$

disect $-\mathrm{K} \% 9.41$ convolve con $1 \ll$ theEnd

Hetpork file: convolve

Height file: $\operatorname{con} 1$

Dimension of input: 1

Dimension $0: 5$

Inputs: 5

Bidden layers: 0

Outputs: 7

Mo. of layers: 2

Layers: 57

Ho. of reights: 42

Enter disection layer neuron ( $D$ to quit):

Dimension of sample: 1

Dinension $0: 6$

Layer: 1

Jeuron: 0

Bias: $-2.22045 e-16$
1.0000
0.0000
0.0000
0.0000
0.0000

Layer neuron ( $D$ to quit):

Dimension of sample: 1

Dimension $0: 5$

Layer: 1

Meuron: 1

Bias: $-6.418480-17$

$$
\begin{array}{lllll}
-1.0000 & 1.0000 & 0.0000 & 0.0000 & 0.0000
\end{array}
$$

Layer a neuron ( $-D$ to quit):

Dimension of sample: 1

Dimension $0: 5$

Lajer: 1

Meuron: 2

Bias: $-1.66533 \bullet-16$

$$
\begin{array}{llllll}
1.0000 & -1.0000 & 1.0000 & 0.0000 & 0.0000
\end{array}
$$

Layer nouron ( $D$ to quit):

Dinension of saple: 1

Dimension $0: 5$

Layer: 1

Iouron: 3

Bias: $2.775560-16$ 


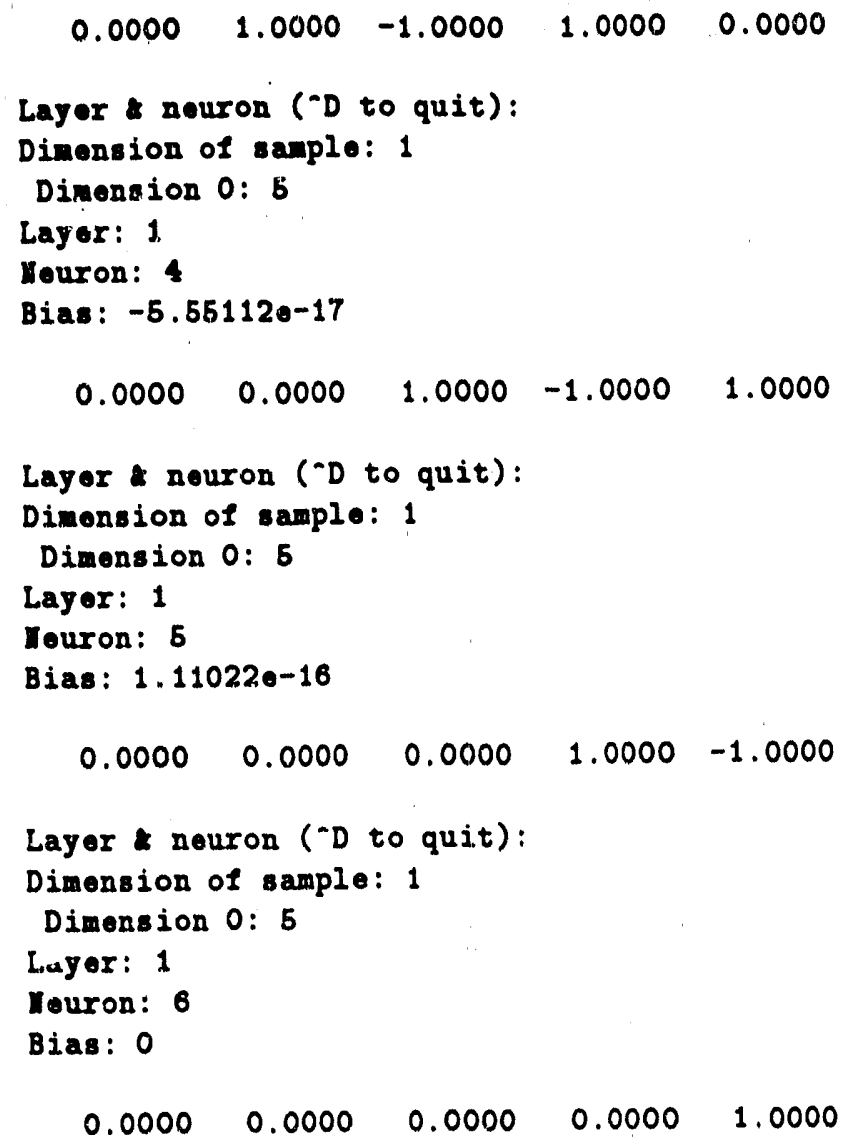

\section{Mathematica Results}

This is the Mathematica ${ }^{\mathrm{TM}}$ script used in computing the various results:

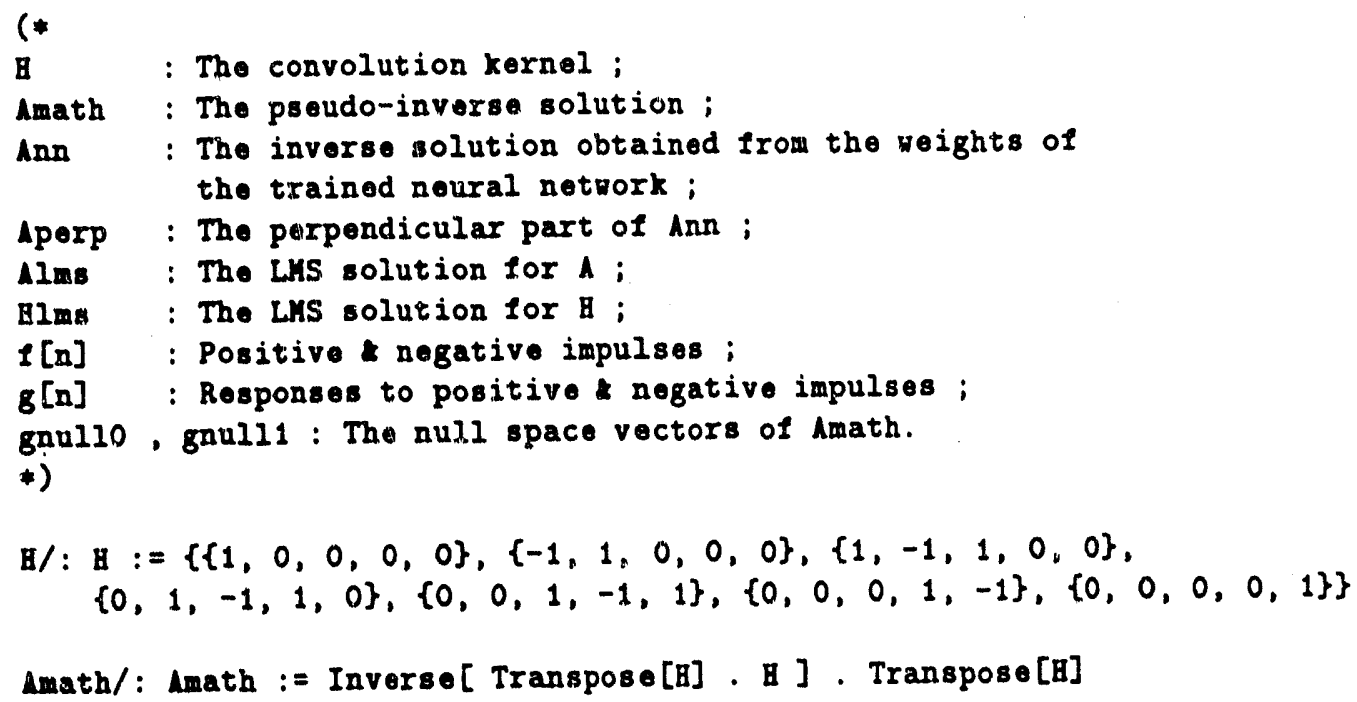




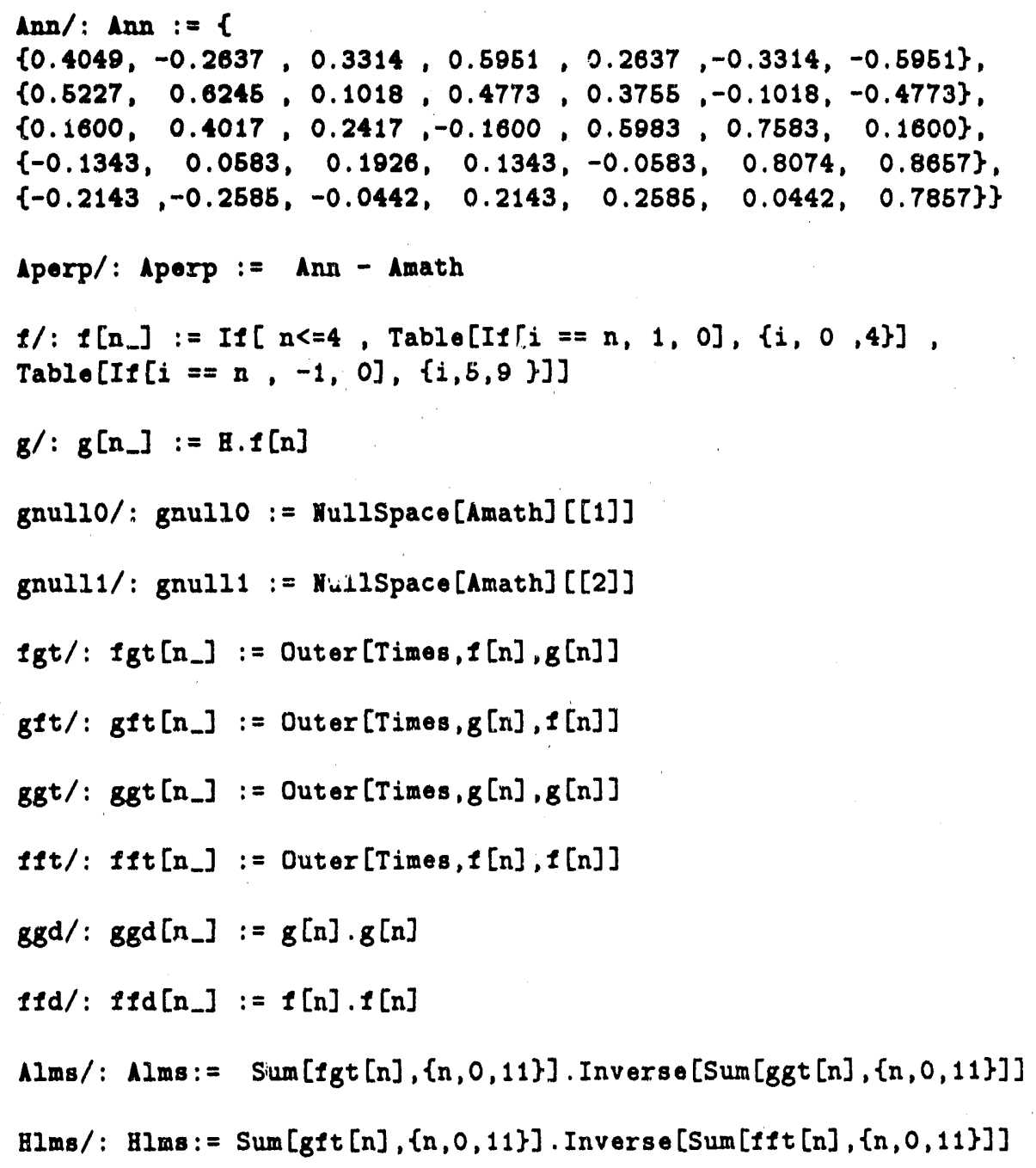

This is a typescript showing the Mathematica ${ }^{\mathrm{TM}}$ results:

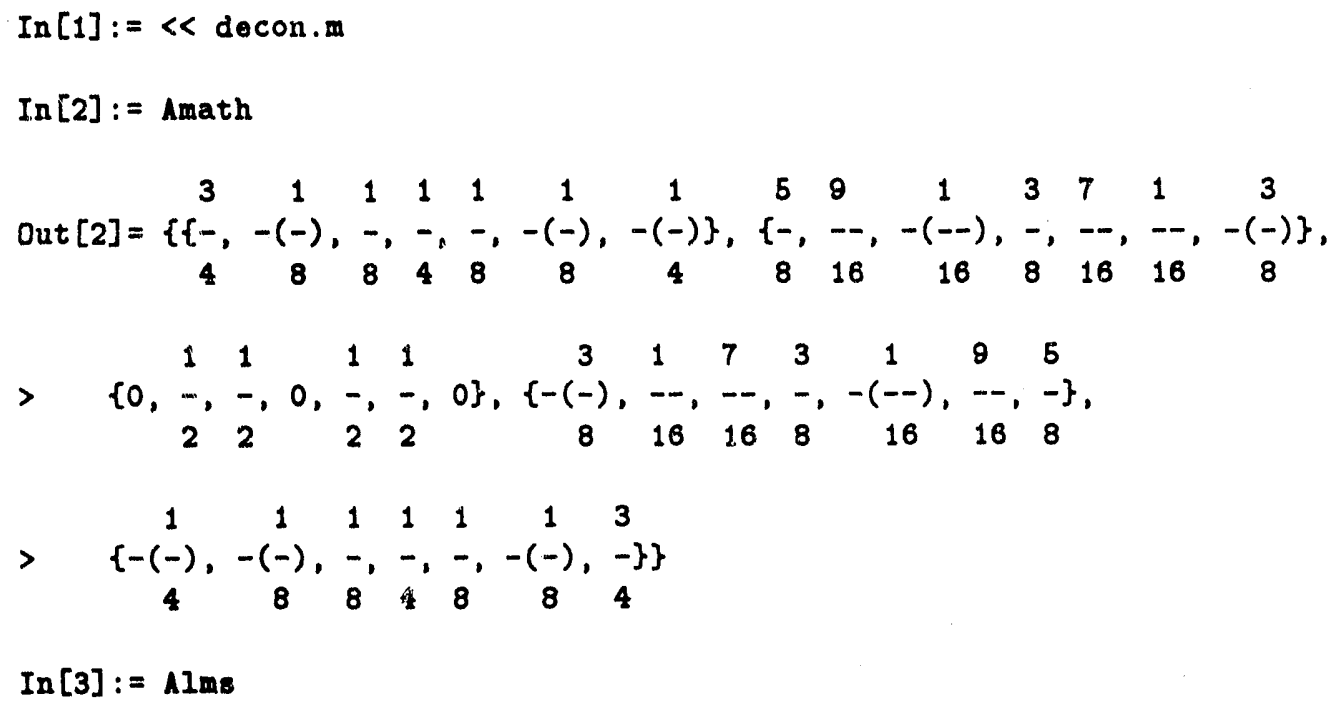




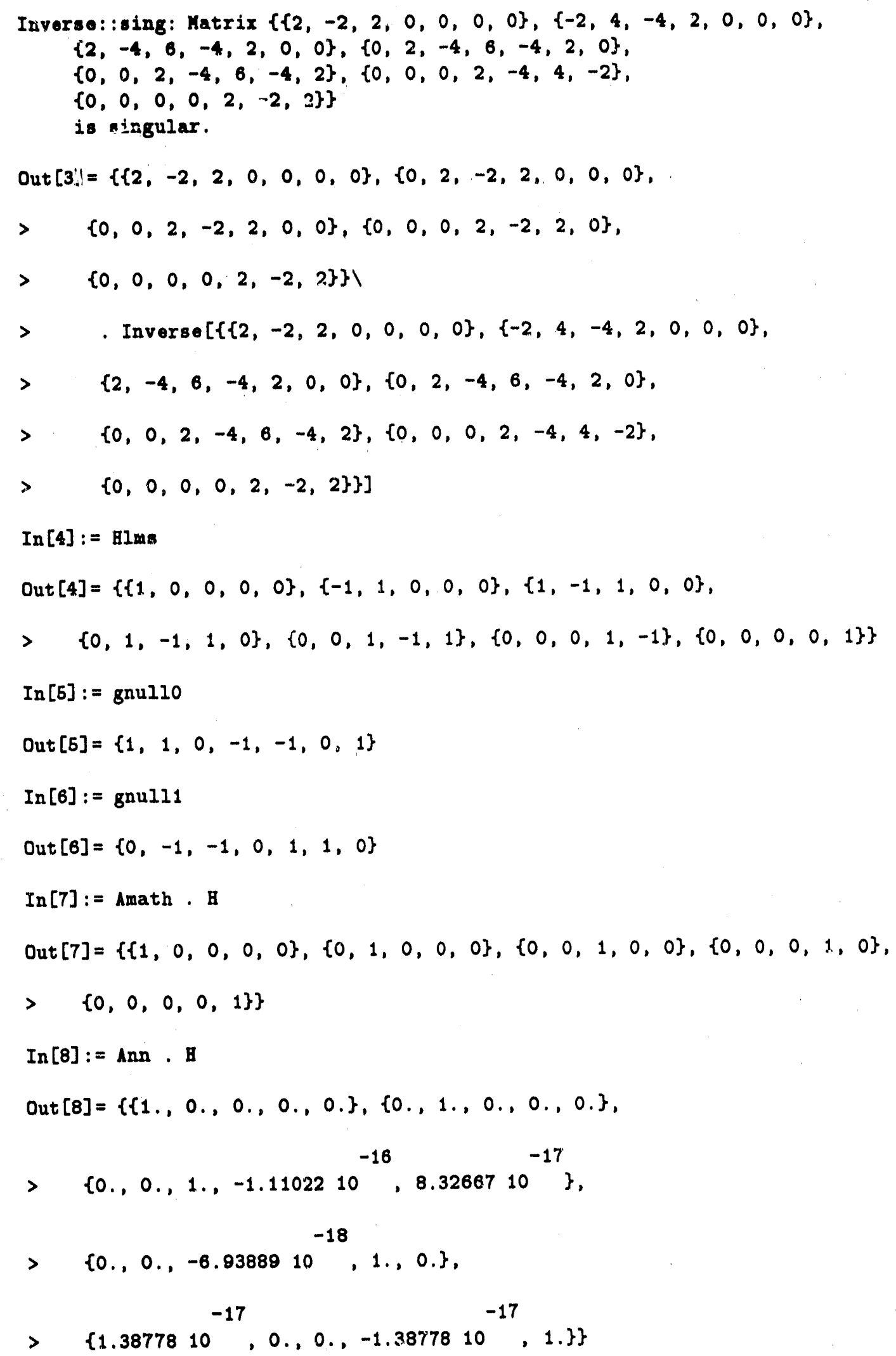




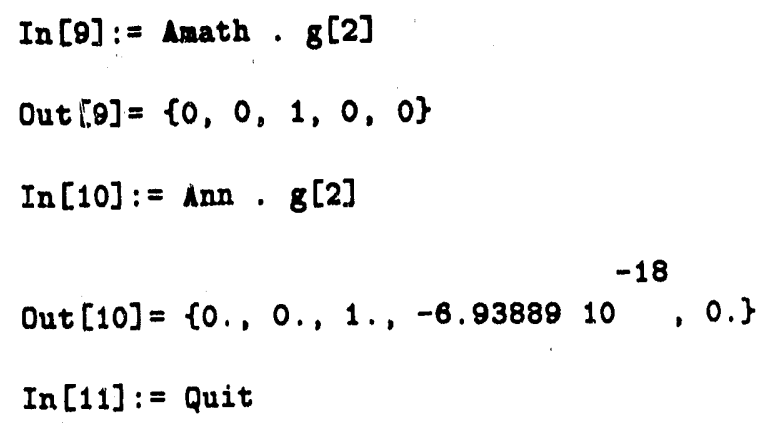


-END-

DATE FILMED

I 13 
1 